\title{
La norma ISO 9001 en el proceso de capacitación en una compañía de servicios petroleros
}

\author{
Norm ISO 9001 in the process of qualification in an oil maintenance \\ company
}
Washington Edy Santillán Marroquín. ${ }^{1}$, Maria Cristina Araque Salazar. ${ }^{2}$ \& Diocelina Guanochanga Oña. ${ }^{3}$

\begin{abstract}
DOI: https://doi.org/10.33262/concienciadigital.v4i2.1.1705

Introduction. The dynamics of human talent processes in modern organizations make them increasingly professional, through the contribution and contribution of quality systems such as ISO 9001. In the training process, apply the standards of this quality standard It is a real challenge for human management leaders, since application, evaluation and monitoring tools must be considered in this process. Objective. Evaluate the impact of the "Training Plan" on the Company, through the application of the ISO 9001 Standard, to demonstrate its application, evaluation, return on investment and the effective monitoring of training in its employees. Methodology. The research design was in the field, the data is collected at the job site. The sample consisted of 10 full-time workers from the administrative, financial, accounting and human talent departments, surveys and interviews were conducted to collect data that consisted of 5 items, it was validated by criteria of specialists in human talent, qualifying validity, relevance and consistency. Results. Among the most frequent results, 75\% know the ISO 9001 standard and its effectiveness in human talent processes $(87.5 \%)$, recognize and validate its effectiveness in the training process $(100 \%)$, show that the standard ISO 9001 in

1 Coordinador Académico del Instituto Superior Tecnológico Bernardo O’Higgins coordinacion.academica@instituto-ohiggins.com, https://orcid.org/0000-0003-1980- 6832, QuitoEcuador

2 Docente del Instituto Superior Tecnológico Bernardo O’Higgins, mcass2008@hotmail.com, https://orcid.org/0000-0002-8425-7289, Quito- Ecuador

3 Docente del Instituto Superior Tecnológico Bernardo O’Higgins, tania.guanochanga@hotmail.com, https://orcid.org/0000-0002-8124-8125, Quito- Ecuador.
\end{abstract}


training, generates motivation, has a positive impact on the work environment and promotes healthy competitiveness. Conclution. It applies in a practical way the concepts of this quality certification with continuous improvement in all its processes, which entails a better interrelation with customers and the best corporate results.

Keywords: ISO 9001 Standard, training, standards, quality, validation.

\section{Resumen.}

Introducción. La dinámica de los procesos de talento humano en las organizaciones modernas, hacen que se profesionalicen cada vez más, mediante el aporte y contribución de los sistemas de calidad como la norma ISO 9001. En el proceso de capacitación aplicar los estándares de ésta norma de calidad, es un verdadero reto para los líderes de la gestión humana, ya que se debe considerar en dicho proceso, herramientas de aplicación, evaluación y seguimiento. Objetivo. Evaluar en la Compañía el impacto del "Plan de Capacitación", mediante la aplicación de la Norma ISO 9001, para evidenciar su aplicación, evaluación, retorno de la inversión y el efectivo seguimiento de la capacitación en los colaboradores de la misma. Metodología. El diseño de investigación fue de campo, los datos son recogidos en el sitio de trabajo. La muestra fue de 10 trabajadores a tiempo completo de los departamentos administrativos, financiero, contable y talento humano, se realizó encuestas y entrevistas de recolección de datos que constó con 5 ítems, fue validado por criterios de especialistas en talento humano, calificando validez, pertinencia y coherencia. Resultados. Entre los resultados con mayor frecuencia, el 75\% conocen la norma ISO 9001 y su efectividad en los procesos de talento humano (87,5\%), reconocen y validan su efectividad en el proceso de capacitación (100\%), evidencian que la norma ISO 9001 en la capacitación, genera motivación, tiene una incidencia positiva en el clima laboral y promueve la sana competitividad. Conclusión. Aplica de manera práctica los conceptos de esta certificación de calidad con el mejoramiento continuo en todos sus procesos, lo que conlleva a una mejor interrelación con los clientes y los mejores resultados corporativos.

Palabras Claves: Norma ISO 9001, capacitación, estándares, calidad, validación.

\section{Introducción.}

La Norma ISO 9000 representa un estándar internacional sobre buenas prácticas de gestión de calidad, promueve la adopción de un enfoque basado en procesos y dentro de la organización conlleva a la identificación de estos procesos y su vinculación a los mismos. Así mismo, se debe determinar cómo debe ser su gestión para producir el resultado esperado.

Un enfoque de esta naturaleza, en el marco de la implementación de un sistema de gestión de la calidad apoya en los siguientes campos: comprender y satisfacer los 
requisitos de los clientes del servicio, considerar los procesos analizando su aporte de valor, eliminando actividades innecesarias, re-procesos que se superponen o que desvían el resultado esperado y finalmente, obtener mediciones del desempeño del proceso de capacitación y sus resultados.

La norma ISO 9001 alineada con la gestión del talento humano, analiza y administra todos aquellos aspectos relacionados con la educación, formación, habilidades, competencias, conocimientos y experiencia de los colaboradores de la organización, consecuentemente da la importancia necesaria a la formación integral (académicatécnica) del personal, en relación al trabajo que realiza y su efecto directo o indirecto a la conformidad de los estándares del producto, con esto determina fomentar una cultura de empresa hacia la calidad y que tenga como base la colaboración entre los trabajadores con todos los niveles de la organización

Consecuentemente, el proceso de la capacitación es relevante en la consolidación de la gestión y el desempeño de sus trabajadores, es la manera de incorporar a la tarea, responsabilidades, las capacidades o habilidades de las personas y generar espacios para el desarrollo de las competencias y sus efectos positivos en la compañía.

\section{Objetivo General.}

Evaluar en la Compañía, el impacto del "Plan de Capacitación", mediante la aplicación de la Norma ISO 9001, para profesionalizar y evidenciar su aplicación, evaluación, retorno de la inversión y el efectivo seguimiento de la capacitación en los colaboradores de la misma.

\section{Objetivos Específicos.}

- Evidenciar el aporte de la Norma ISO 9001 en el plan de capacitación, que permita demostrar la integralidad del sistema en la empresa.

- Demostrar que los indicadores de gestión, basados en el Sistema de Gestión de Calidad, son los adecuados para la medición de la efectividad del programa de Capacitación de la empresa.

- Observar la pertinencia e importancia de la implantación del Plan de Capacitación en la empresa, para determinar su efecto en la gestión del personal y en la satisfacción de los clientes.

\section{Metodologia.}

Para esta investigación la metodología que se utilizó es la de campo. Según Castillo, (2005) Los datos son recogidos directamente del sitio donde se encuentra el objeto de estudio, es una investigación "in situ", directamente recogida de los trabajadores que laboran en el campo administrativo de la empresa.

Se aplicará una deducción que va en la parte general que emplea toda la norma a los casos particulares de las áreas administrativas, finanzas y talento humano. Según E- 
educativa Cat Edu (2011). El método inductivo intenta ordenar la observación tratando de extraer conclusiones de carácter universal desde la acumulación de datos particulares.

La población investigada es de 10 colaboradores, los cuales se desempeñan en calidad de trabajadores a tiempo completo, entre principales, delegada de la norma ISO 9001, responsable del plan de capacitación, departamento de contabilidad y departamento de talento humano de la empresa de servicios petroleros.

La selección de la muestra para la presente investigación será de tipo aleatoria simple. Según Casal \& Mateu (2003) en el muestreo aleatorio simple se define que todas las personas tienen la misma probabilidad de ser elegidos para concretar el tamaño de la muestra y se extraen al azar los elementos investigados. El presente estudio tendrá como propósito fundamental el de conocer si existe un gran impacto de calidad entre la norma ISO 9001 y el plan de capacitación en la empresa de servicios petroleros.

La importancia de la capacitación radica especialmente en fortalecer el desempeño laboral de los trabajadores y esto a su vez permite la mejora continua en la productividad de los capacitados lo cual se traducirá en la reducción de costos e incremento de rentabilidad, apoyándose en la norma ISO 9001 y la respectiva sistematización de los datos obtenidos.

\section{Criterios de capacitación y desempeño con la norma ISO 9001}

El proceso de capacitación le permite a la compañía definir y diagnosticar las necesidades reales de formación, entrenamiento y educación para el personal a fin de buscar una productividad eficiente que promueva la calidad como indicador. A través de la evidencia de las carencias propias de cada puesto de acuerdo a sus funciones, su nivel jerárquico y su propia autonomía e independencia. Y a su vez la empresa mantendrá un registro ordenado de las capacitaciones dadas.

Las directrices propuestas en la norma ISO 9004 buscan establecer las instrucciones para la implantación de la norma que en su punto 5.2.4. indica lo siguiente: Es la dirección de la organización quien debe precisar los niveles tanto de competencia como de experiencia y capacitación que requiere el personal para garantizar que el personal tenga las capacidades requeridas para un desempeño adecuado. De igual manera el punto 18.1.1. se busca el seguimiento y la documentación de la capacitación para que esta pueda ser debidamente instaurada y mantenida.

La calidad es uno de los parámetros más relevantes para la norma ya que es a través de esta que se puede lograr eficiencia dentro de las organizaciones. Para ello todos los procesos de talento humano deben estar focalizados con este propósito, para que el esfuerzo tenga una ruta consensuada entre el trabajador y la empresa y sus objetivos. 


\section{La norma ISO 9001 y la competencia laboral}

De acuerdo al Equipo Vértice (2010), para definir las normas de calidad bajo el sistema de competencias laborales, estas como tal tendrán una implicación directa en los parámetros de desempeño y a su vez en la parte cognitiva que incluye directamente en los procesos de mejora continua así como, incide en la búsqueda de las organizaciones por rentabilidad y productividad.

Los puntos comunes entre los sistemas de calidad ISO y las competencias laborales radican en la búsqueda de la mejora continua y de la retroalimentación mediante procesos documentados y llevados en forma sostenida en la organización.

\section{El rol de la alta dirección en la compañía}

El compromiso de la alta dirección es uno de los factores claves, ya que es un trabajo en equipo pero por sobre todo se debe conseguir el logro del cumplimiento de los objetivos organizacionales a través de cada una de las etapas de la implementación de la norma ISO 9001, que engloba dentro del proceso al entorno global de la organización; es decir, trabajadores, clientes, proveedores y todo aquel que tenga una relación con la organización entendiendo su responsabilidad y su rol a desempeñar. El liderazgo entonces trazará la ruta mediante la cual se podrá motivar eficientemente al personal y creará un sentimiento compartido en el cual los trabajadores entiendan su papel frente a la competitividad y la productividad.

Alinear las decisiones en este nivel de liderazgo con el sistema de gestión de calidad y que ella misma tenga presente esta estrategia de gestión son los objetivos principales para una mejora continua productividad y rentabilidad de la compañía.

Mostrarse como responsables ante sus potenciales clientes, proveedores, ser cabezas visibles de su propia actividad empresarial y mostrar de manera transparente sus acciones de calidad, son factores claves que refuerzan su liderazgo y buena imagen a nivel social.

El experto en estandarización, Oscar Combs, en su estudio para la Harvard Business School, asegura que "ISO 9001 no solo ofrece beneficios de calidad. Esta norma debería ser concebida como una herramienta de gestión y organización de negocio pensada para obtener valor añadido, mejorar sus procesos y reducir sus riesgos". Sus palabras destacan la importancia de sistematizar y generar protocolos de la calidad total en cualquier proceso y nivel de la compañía.

\section{Ventajas al implementar ISO 9001 en una empresa}

Las Normas ISO a nivel mundial, establecen las directrices que debe cumplir una empresa para garantizar la gestión de calidad de un bien o servicio. Las normas realizan un proceso de recolección de contenido, guías, herramientas y métodos de auditoría a ser implantadas en la empresa. La norma ISO 9001 especifica la manera en que una organización opera sus estándares de calidad, tiempos de entrega, documentos de 
evidencia y niveles de servicio. La implantación de estas normas de calidad es un duro trabajo, pero ofrece numerosas ventajas para las empresas, por ejemplo:

- Estandarizar las actividades del personal que trabaja dentro de la organización por medio de la documentación.

- Incrementar la satisfacción del cliente interno y externo.

- Medir y monitorizar el desempeño de los procesos.

- Disminuir re-procesos.

- Incrementar la eficacia y/o eficiencia de la organización en el logro de sus objetivos alineados al plan estratégico.

- Mejorar continuamente sus procesos, productos, servicios, etc.

- Reducir las incidencias de producción o prestación de servicios.

El talento humano es el eje principal de toda empresa y su influencia para un mejor desarrollo es decisiva. El talento humano es y continuará siendo el activo más valioso de una empresa. Bajo este análisis, la gestión humana, dentro de sus procesos claves está la selección del talento por competencias cada vez más asertiva y luego la capacitación, entrenamiento, formación y desarrollo de su gente.

Los empresarios, líderes, directivos y la alta gerencia de las instituciones, se plantean la necesidad de contar con programas de capacitación y desarrollo que promuevan el crecimiento del talento humano que permitan el incremento de la productividad, rentabilidad y calidad en el desempeño de las tareas y responsabilidades laborales.

\section{Plan de capacitación}

Según Barbies, (2000) el plan de capacitación es una guía que prepara e integra varios pasos a seguir en la formación, entrenamiento y desarrollo del personal, con el objetivo de brindar conocimientos, desarrollo de competencias y actitudes necesarias para su realización integral. La capacitación en la empresa permite a los colaboradores brindar lo mejor de sus aportes para un desempeño eficiente y eficaz en su cargo.

\section{Norma ISO 9001}

Según (Calavia, 2011) es una norma de sistema de gestión de calidad, que aporta para gestionar y controlar de manera continua la calidad en todos los procesos. Apoyando a las organizaciones a mejorar su rendimiento y conducirles hacia la eficiencia, servicio al cliente y excelencia en el producto o servicio.

\section{Resultados}

Para evaluar la aplicabilidad de la variable "Norma ISO 9001 y su impacto en el plan de capacitación", se aplicó encuestas diferenciadas, de modo que se seleccionaron a varios trabajadores de cada área, las mismas contenían respuestas cerradas y abiertas, que luego se tabularon para finalmente interpretar los resultados. 
En términos generales, se observó un alto nivel, de casos que poseen criterio y conocimiento en la aplicación de la Norma ISO 9001 en todos los procesos en los que se encuentran inmersos las áreas investigadas; además, que en base a lo mencionado, existió un fuerte sustento para gestionar el "Plan de Capacitación" de la empresa, lo cual constituye una herramienta poderoso para la aplicación de procesos, la satisfacción de sus clientes y el manejo de un proceso coordinado de mejora continua.

\section{Delegados de la implementación de la norma ISO 9001}

1. Se socializó a todo el personal los criterios para la implementación de la norma ISO 9001 en la producción de bienes o servicios

2. Se determinaron las herramientas necesarias para la aplicación de la norma.

3. Se acuerda la participación en el sistema de gestión de la calidad en el desarrollo del plan integral de capacitación de la empresa

4. Los indicadores de gestión de la empresa, permiten medir el impacto del plan de capacitación de la empresa, bajo la influencia de la Norma ISO 9001.

5. Existe cierto tipo de retroalimentación por parte de los empleados de la empresa, para rediseñar el plan de capacitación netamente enmarcado al sistema de gestión de la calidad.

Documento No. 1 (Síntesis de las respuestas obtenidas en la investigación)

1. Para alcanzar el debido nivel de compromiso con respecto a la norma, la debida sensibilización y su cumplimiento sea fácil y más conocido.

2. Si, software, capacitación, sensibilización / campañas

3. Si, es parte de la estrategia el contar con el apoyo del talento humano

4. Si, son medidas y seguimiento del BSC (balance score card)

5. Si, evaluación del desempeño, reporte de hallazgos (No conformidades)

TABULACION ENCUESTA A DELEGADOS DE LA IMPLEMENTACION DE LA NORMA

\begin{tabular}{|c|c|c|c|}
\hline PREGUNTA & Colaborador 1 & $\% \mathrm{SI}$ & $\%$ NO \\
\hline $\begin{array}{l}\text { 1. Se socializo a todo el personal los preceptos de la } \\
\text { implementación de la norma ISO para la producción de } \\
\text { bienes o servicios. }\end{array}$ & SI & $100 \%$ & $0 \%$ \\
\hline $\begin{array}{l}\text { 2. Se establecieron las herramientas necesarias para la } \\
\text { aplicación de la norma. }\end{array}$ & SI & $100 \%$ & $0 \%$ \\
\hline $\begin{array}{l}\text { 3. Se estipula con claridad el aporte del sistema de } \\
\text { gestión de la calidad en el desarrollo del plan integral } \\
\text { de capacitación de la empresa. }\end{array}$ & SI & $100 \%$ & $0 \%$ \\
\hline $\begin{array}{l}\text { 4. Los indicadores de gestión de la empresa, permiten } \\
\text { medir el impacto del plan de capacitación de la } \\
\text { empresa, bajo la influencia de la ISO } 9001 \text {. }\end{array}$ & SI & $100 \%$ & $0 \%$ \\
\hline
\end{tabular}


5. Existe algún tipo de retroalimentación de los servidores de la empresa, que permita rediseñar el plan de capacitación alineado al sistema de gestión de la calidad.

Tabla No. 1 Encuesta a delegados de la implementación de la norma

Elaborado por: Los autores

\section{TABULACION ENCUESTA A DELEGADOS DE LA IMPLEMENTACION DE LA NORMA}

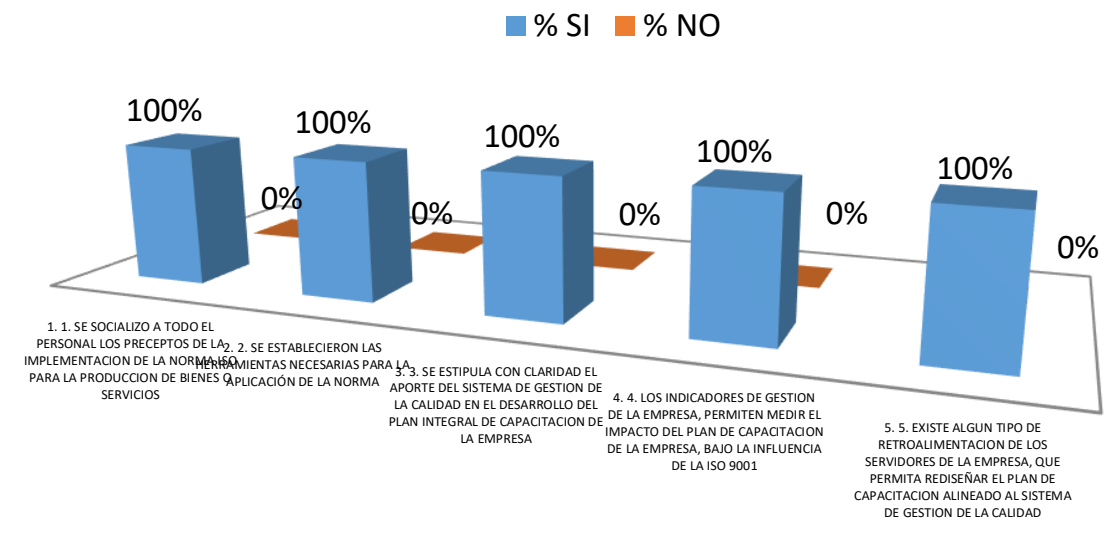

Figura No. 1 Encuesta a delegados de la implementacion de la norma

Elaborado por: Los autores

\section{Responsables del proceso de capacitación de la empresa}

1. ¿Al elaborar el plan integral de capacitación de la empresa, qué aspectos de la norma se consideraron para su desarrollo?

2. ¿El plan integral de capacitación responde a las exigencias del sistema de gestión de la calidad?

3. ¿Para el diseño del plan integral de capacitación se consideraron los indicadores de gestión que contempla la evaluación de su efectividad?

4. ¿Existe algunos factores en los que se pueda relacionar la efectividad del plan integral de capacitación y la satisfacción de los clientes?

5. ¿La implementación del plan integral de capacitación alineado con las exigencias del sistema de gestión de la calidad tiene efectos en el clima laboral de la empresa?

Documento No. 02 (Síntesis de las respuestas obtenidas en la investigación)

1. Documentación y trazabilidad de requerimientos a fin de gestionar el desarrollo de los colaboradores y de los procesos.

2. El plan integral de capacitación se establece en base a los perfiles de cada cargo.

3. Se realiza la medición de la efectividad del curso según al proceso al que se aplica. 
4. Son dos procesos que se manejan por separado, en la evaluación al desempeño, se consideran aspectos relacionados a la capacitación propiamente, mientras que en la evaluación de "atención al cliente" (interno y externo) se consideran los factores de satisfacción al cliente.

5. Sí, porque los colaboradores lo toman como un beneficio para ellos.

\begin{tabular}{|c|c|c|c|}
\hline \multicolumn{4}{|c|}{$\begin{array}{l}\text { ENCUESTA A RESPONSABLES DEL PROCESO DE CAPACITACION DE LA } \\
\text { EMPRESA }\end{array}$} \\
\hline PREGUNTA & Colaborador 1 & $\% \mathrm{SI}$ & $\% \mathrm{NO}$ \\
\hline $\begin{array}{l}\text { 1. ¿Al elaborar el plan integral de capacitación de la } \\
\text { empresa, qué aspectos de la norma se consideraron } \\
\text { para su desarrollo? }\end{array}$ & SI & $100 \%$ & $0 \%$ \\
\hline $\begin{array}{l}\text { 2. ¿El plan integral de capacitación responde a las } \\
\text { exigencias del sistema de gestión de la calidad? }\end{array}$ & NO & $0 \%$ & $100 \%$ \\
\hline $\begin{array}{l}\text { 3. ¿Para el diseño del plan integral de capacitación se } \\
\text { consideraron los indicadores de gestión que } \\
\text { contempla la evaluación de su efectividad? }\end{array}$ & $\mathrm{NO}$ & $0 \%$ & $100 \%$ \\
\hline $\begin{array}{l}\text { 4. ¿Existe algunos factores en los que se pueda } \\
\text { relacionar la efectividad del plan integral de } \\
\text { capacitación y la satisfacción de los clientes? }\end{array}$ & $\mathrm{NO}$ & $0 \%$ & $100 \%$ \\
\hline $\begin{array}{l}\text { 5. ¿La implementación del plan integral de } \\
\text { capacitación alineado con las exigencias del sistema } \\
\text { de gestión de la calidad tiene efectos en el clima } \\
\text { laboral de la empresa? }\end{array}$ & SI & $100 \%$ & $0 \%$ \\
\hline
\end{tabular}

Tabla No. 2 Encuesta a responsables del proceso de capacitacion de la empresa

Elaborado por: Los autores

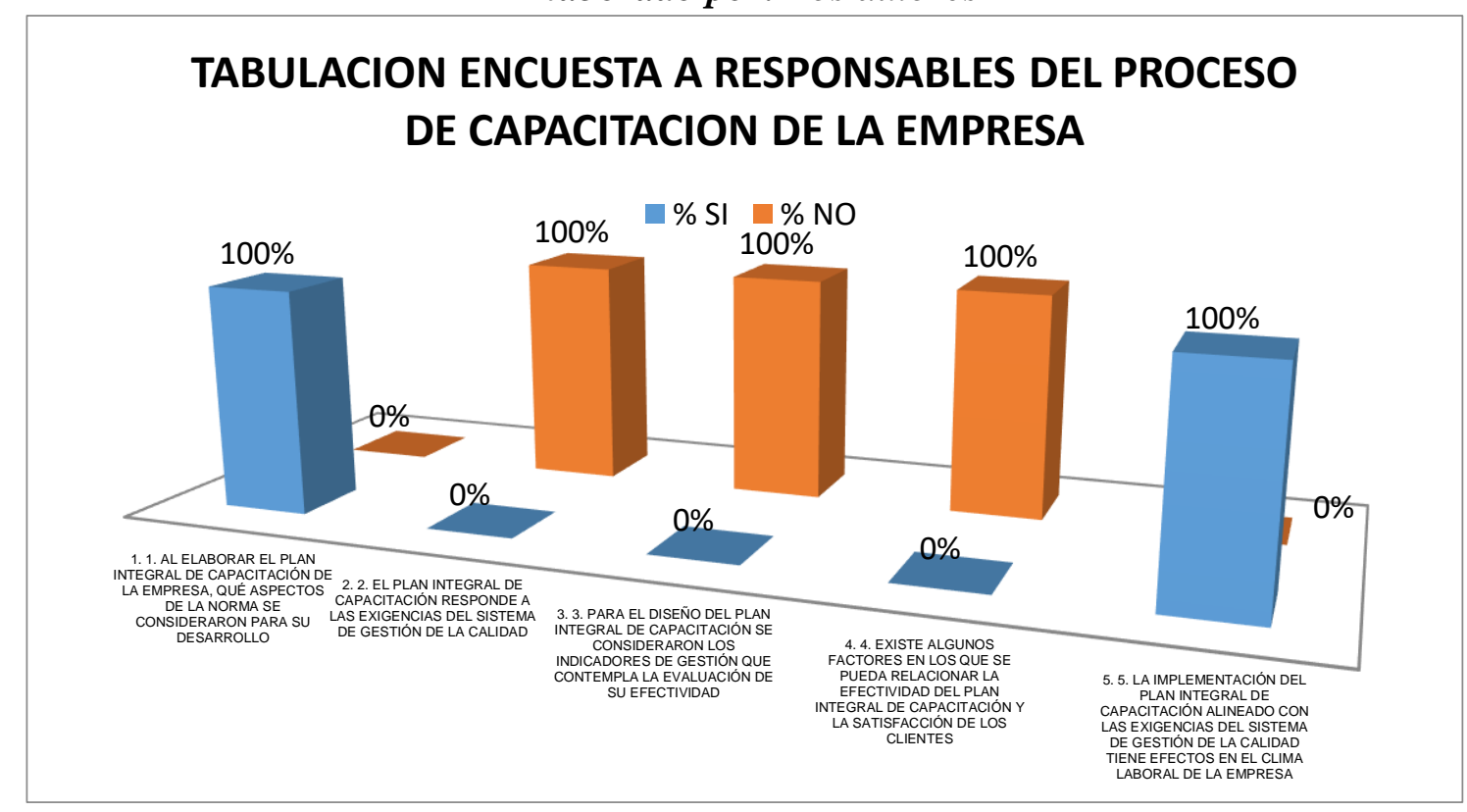

Figura no. 2 Encuesta a responsables del proceso de capacitacion de la empresa

Elaborado por: Los autores 
ENTREVISTA CON LOS COLABORADORES DEL DEPARTAMENTO DE FINANCIERO- CONTABLE

1. ¿Conoce con exactitud los objetivos y el alcance del sistema de gestión de la calidad ISO 9001 ?

2. ¿Usted cree que exista una relación entre el sistema de gestión de la calidad y el plan integral de capacitación?

3. ¿Podría aseverar, si existe en la empresa un mejoramiento continuo en la capacitación, relativa a los procesos técnicos y administrativos?

4. Los indicadores de gestión de la empresa permiten evaluar de mejor manera la eficiencia del plan integral de capacitación.

5. La aplicación del sistema de gestión de calidad ha permitido mejorar la motivación y el clima laboral de la empresa y realmente es una ventaja competitiva frente a la competencia.

Documento No. 03 (Síntesis de las respuestas obtenidas en la investigación)

1. Si, nuestra empresa tiene y trabaja bajo esta y otras certificaciones de calidad.

2. Si, por cuanto toda la organización debe estar alineada a alcanzar la calidad en sus procesos.

3. Sí, es por política de la empresa mantener constantes capacitaciones especialmente en el personal técnico.

4. $\mathrm{Si}$, permiten mejorar en las áreas donde requieren de una mayor inducción y capacitación.

5. $\mathrm{Si}$, mientras las empresas tengan por objetivo mejoras continuas en sus procesos de mejora y llegar a la excelencia.

Documento No. 04 (Síntesis de las respuestas obtenidas en la investigación)

1. No, no he tenido la necesidad de acuerdo al puesto de trabajo

2. Si, son dos actividades relacionadas en una organización.

3. No, no existe capacitación.

4. Si, estos indicadores permiten analizar las debilidades del personal y mejorar con capacitación.

5. Si, realmente permite mejorar las relaciones ideales entre el personal.

Documento No. 05 (Síntesis de las respuestas obtenidas en la investigación)

1. Si, trata sobre la calidad y satisfacción del cliente

2. Si, ya que un personal capacitado puede brindar un servicio de calidad y lograr la satisfacción del cliente.

3. Si, existe un proceso de capacitación en el área técnica administrativa pero falta más.

4. Si, permite evaluar los indicadores que la empresa necesita para evaluar la eficiencia del Plan Integral de Capacitación. 
5. Si, la gestión de la calidad y un Plan de Capacitación continuo es lo que ha logrado a esta empresa crecer, y esto ha permitido ser de competencia para otras empresas extranjeras que proporcionar un similar servicio.

Documento No. 06 (Síntesis de las respuestas obtenidas en la investigación)

1. La ISO 9001, controla todos los procesos de una organización realizadas con calidad y eficiencia en beneficio de todos los que conforman una empresa.

2. No responde

3. Si, existe una planificación acerca del proceso de capacitación, permite que el colaborador desempeñe de mejor manera sus actividades.

4. Se pretende alcanzar el $100 \%$ de cumplimiento en el aspecto de capacitación, para ello se plantea lineamientos a seguir.

5. La parte más importante de una organización es su talento humano y ganarse el compromiso de los mismos, hará que la organización tenga una ventaja competitiva.

Documento No. 07 (Síntesis de las respuestas obtenidas en la investigación)

1. Sí, son procesos necesarios para el Sistema de Calidad.

2. Sí, claro porque al tener un Plan Integral de Capacitación se logra calidad total.

3. No

4. Sí, siempre y cuando se tomen las medidas de mejora, después de haber obtenido los indicadores.

5. Pienso que sí, hay ciertos aspectos que dependen mucho del trabajador como tal.

Documento No. 08 (Síntesis de las respuestas obtenidas en la investigación)

1. Si, ISO 9001 abarca todo el proceso que interviene en la prestación o fabricación de un bien, garantizando el producto final.

2. Sí, porque para tener un eficiente sistema de calidad se debe capacitar a todo el personal que interviene en el proceso, así cuenten con el conocimiento y la experiencia que se requiere.

3. Si, la empresa en su Plan de Capacitación, recolecta por áreas y personas los temas que se requieren capacitar para realizar sus actividades de una forma efectiva y eficiente, con esto se mejora y optimiza los procesos.

4. Si, ya que los indicadores representan las actividades y la forma en cómo se hace el trabajo, si los indicadores son favorables se entendería que el plan de capacitación si cumple su función.

5. Sí, porque con un SGC (sistema de gestión de calidad) cada persona conoce las actividades que le corresponden hacer, como se deben ejecutar, esto hace que el proceso se ágil y eficiente, lo que conlleva una ventaja con la competencia y una respuesta al cliente más rápida.

Documento No. 09 (Síntesis de las respuestas obtenidas en la investigación)

Para los colaboradores de Talento Humano 
1. Si, incrementa la satisfacción del cliente, mediante la mejora continua

2. Si, la capacitación continua del personal, es directamente proporcional con la calidad y el sistema de gestión.

3. Si, la escuela de entrenamiento en el área de operaciones

4. Si, evaluaciones al personal de horas hombre y capacitaciones anuales.

5. Si, en la encuesta de clima laboral

6. Si, la escuela de entrenamiento en el área de operaciones

7. Si, evaluaciones al personal de horas hombre y capacitaciones anuales.

8. Si, en la encuesta de clima laboral.

ENCUESTA A LOS COLABORADORES DEL DEPARTAMENTO FINANCIEROCONTABILIDAD Y DE TALENTO HUMANO

\begin{tabular}{|c|c|c|c|c|c|c|c|c|c|c|c|c|}
\hline PREGUNTA & 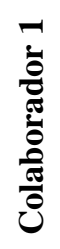 & 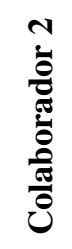 & 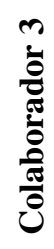 & 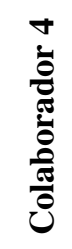 & n & $\begin{array}{l}b \\
\dot{0} \\
\frac{\sigma}{0} \\
\frac{0}{0} \\
\frac{\sigma}{0} \\
\dot{0}\end{array}$ & 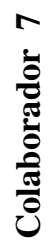 & $\begin{array}{l}\infty \\
\dot{0} \\
\frac{0}{0} \\
\frac{0}{0} \\
\frac{0}{0} \\
\dot{0}\end{array}$ & $\sum_{\infty}^{\infty}$ & 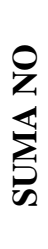 & $\begin{array}{l}\text { न } \\
\text { a }\end{array}$ & $\begin{array}{l}\vartheta_{Z} \\
\text { z } \\
0\end{array}$ \\
\hline $\begin{array}{l}\text { 1. ¿Conoce con } \\
\text { exactitud los } \\
\text { objetivos y el alcance } \\
\text { del sistema de gestión } \\
\text { de la calidad ISO } \\
9001 ?\end{array}$ & SI & $\mathrm{NO}$ & SI & SI & SI & SI & SI & SI & 7 & 1 & $87,5 \%$ & $12,5 \%$ \\
\hline $\begin{array}{l}\text { 2. ¿Usted cree que } \\
\text { exista una relación } \\
\text { entre el sistema de } \\
\text { gestión de la calidad } \\
\text { y el plan integral de } \\
\text { capacitación? }\end{array}$ & SI & SI & SI & $\mathrm{NO}$ & SI & SI & SI & SI & 7 & 1 & $87,5 \%$ & $12,5 \%$ \\
\hline $\begin{array}{l}\text { 3. ¿Podría aseverar, si } \\
\text { existe en la empresa } \\
\text { un mejoramiento }\end{array}$ & & & & & & & & & & & & \\
\hline $\begin{array}{lr}\text { continuo en la } \\
\text { capacitación, } & \text { relativa } \\
\text { a los } & \text { procesos }\end{array}$ & SI & NO & SI & SI & NO & SI & SI & SI & 6 & 2 & $75,0 \%$ & $25,0 \%$ \\
\hline $\begin{array}{ll}\text { técnicos } & \mathrm{y} \\
\text { administrativos? } & \end{array}$ & & & & & & & & & & & & \\
\hline $\begin{array}{l}\text { 4. Los indicadores de } \\
\text { gestión de la empresa } \\
\text { permiten evaluar de } \\
\text { mejor manera la } \\
\text { eficiencia del plan } \\
\text { integral } \\
\text { capacitación. }\end{array}$ & SI & SI & SI & $\mathrm{NO}$ & SI & SI & SI & SI & 7 & 1 & $87,5 \%$ & $12,5 \%$ \\
\hline
\end{tabular}


5. La aplicación del sistema de gestión de calidad ha permitido mejorar la motivación y el clima laboral de la empresa $\mathrm{y}$

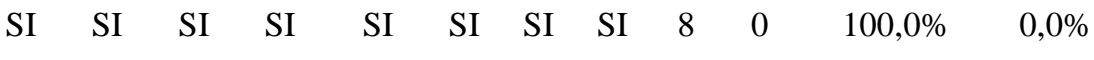
realmente es una ventaja competitiva frente a la competencia.

Tabla No. 3 Encuesta a los colaboradores del departamento financiero- contabilidad y de talento humano

\section{Elaborado por: Los autores}

Documento No. 10 (Síntesis de las respuestas obtenidas en la investigación)

Para los colaboradores de Talento Humano

1. Si, genera gran impacto.

2. Sí, porque está orientado al mejoramiento continuo en los diferentes procesos de la organización teniendo repercusión en la productividad del personal - ligado a capacitación y desarrollo.

3. Sí, porque se incluyen anualmente en el plan de capacitación, cursos orientados a formar al personal en el conocimiento, competencias y manejo de normas de calidad.

4. Sí, porque se ha dado prioridad a la formación en estos temas por los resultados obtenidos.

5. Si, actualmente el personal lo percibe como un beneficio en toda la organización.

TABULACION ENCUESTA A CONTABILIDAD Y

TALENTO HUMANO

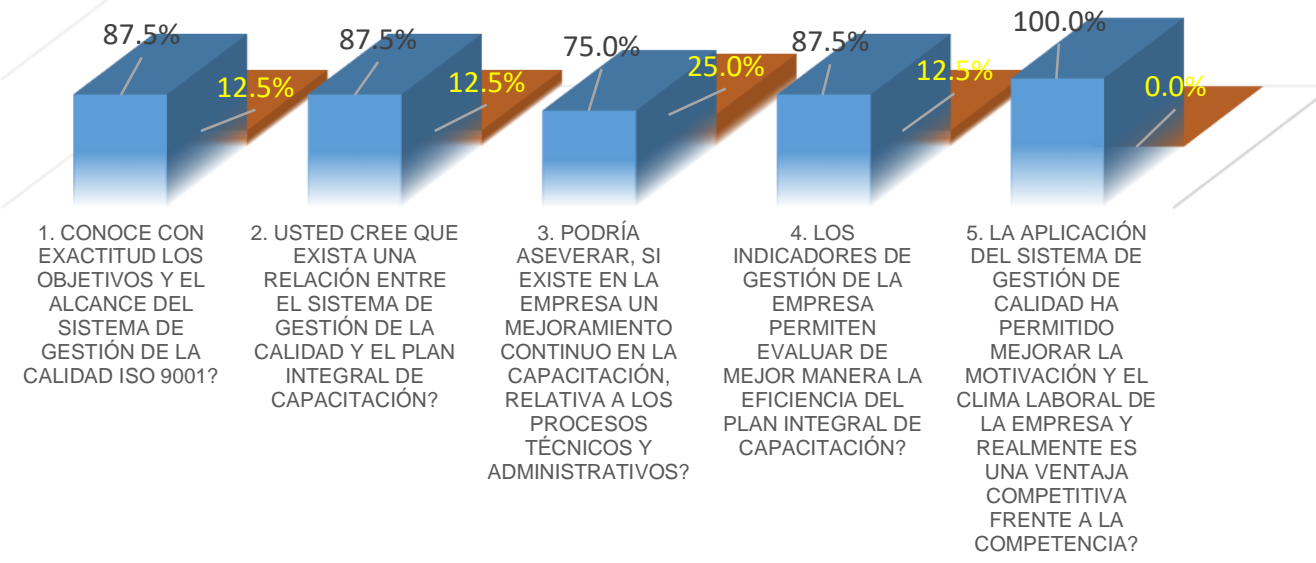

Figura No. 3 Tabulación encuesta a los departamentos financiero- contable y talento humano

Elaborado por: Los autores 


\section{Presentación y análisis de resultados}

Las normas ISO proporcionan un sistema compresivo de las reglas y regulaciones, especificaciones y recomendaciones objetivas que le permiten a una empresa, grande, mediana o pequeña, para establecer procesos y procedimientos de calidad realizables y así poder funcionar sistémicamente de manera eficiente y eficaz.

La norma ISO 9001 considera que un trabajador es competente cuando cumple los requisitos de educación (estudios mínimos que una persona debe tener para un determinado puesto), formación (refiere específicamente como aquellos conocimientos adicionales que son necesarios para desempeñar las actividades de un puesto), competencias (de tipo práctico como por ejemplo la habilidad y buena percepción ya sea ocular u olfativa, que debe tener un vendedor, técnico, administrativo, etc.) y experiencia que la organización determina para cada puesto de trabajo (especifica la que el trabajador debe tener en el puesto o en puestos similares incluido un período de prácticas mínimo en la empresa).

Estas competencias son las mínimas que debe tener una persona para realizar su trabajo con éxito y que van a diferenciar a los trabajadores con desempeño excelente de los que tienen un desempeño normal."

De esta manera, se determina que la aplicación de la norma ISO 9001 proporciona una serie de factores que permiten fundamentar el diseño del "Plan Integral de Capacitación" para la generación de mayor valor agregado en la formación del personal y por lo tanto en una ventaja competitiva frente a empresas similares del sector, así mismo, posibilita una mayor satisfacción en el trabajador, ya que encamina los objetivos personales a los corporativos que es una herramienta integral, medible y verificable en el camino del mejoramiento continuo, y con ello, la satisfacción de los clientes.

\section{Conclusiones}

- Considerando las fuentes primarias y secundarias de información, y la implementación de la ISO 9001 es una norma internacional de calidad que toma en cuenta las actividades de una organización y por ende las de talento humano. Esta norma se concentra en la satisfacción del cliente y en la capacidad y formación de proveer productos y servicios que cumplan con los requerimientos internos y externos de la organización. En este caso en particular la empresa de servicios petroleros visiona la aplicación de la norma ISO 9001 en todas las áreas de la empresa y en especial en la formación y capacitación del personal, para el cual determina que un personal motivado aplica los conceptos y preceptos de esta certificación de calidad con el mejoramiento continuo en todos sus procesos, lo que conlleva a una mejor interrelación con los clientes y los mejores resultados corporativos.

- Considerando la modalidad de esta investigación, se determinó que con la implementación del Sistema de Gestión de la Calidad, se observa la pertinencia 
e importancia del Plan de Capacitación en la empresa de servicios petroleros, ya que tiene su efecto en la gestión del personal, en la asignación de los recursos, control de riesgos asociados, control de costos, define y aplica una política de calidad organizacional que considera las necesidades y expectativas de los clientes internos y externos con mejoras tangibles y la consideración de la visión de futuro de la organización que siendo del entorno de la explotación de los hidrocarburos, incrementará notablemente los estándares de calidad en todos sus procesos ya que son de mayor exigencia.

- Un sistema de calidad genera un personal de calidad. El talento humano se considera de calidad, cuando tiene un valor agregado en cuatro aspectos: educación, formación, competencias y experiencia; además, cuando está consciente de la importancia de sus actividades en relación con la calidad, y cuando está satisfecho, situación que se observó en este estudio que se puede medir y comprobar en el clima laboral de la empresa. En este aspecto, y con la tabulación de las encuestas realizadas en la empresa, se determinó que los colaboradores del departamento de Talento Humano, elabora de forma metódica el Plan de Capacitación para su personal teniendo como baso el sólido sistema integrado de gestión de Calidad, Ambiente, Salud y Seguridad Ocupacional que acredita trabajar bajo normas: ISO 9001, ISO 14001; OHSAS 18001, Norma API Q1 y Q2 del American Petroleum Institute y estampe ASME, además certifican que su planta de producción están en la capacidad de producir y diseñar cualquier tipo de producto o equipo para las industrias energéticas.

- Los métodos de investigación utilizados, determinaron que los indicadores de gestión muestran la estrecha relación entre la administración de la empresa y la satisfacción de los clientes, esto se demuestra en el continuo crecimiento de la empresa desde su creación en el año 1990 y su internacionalización; además, actualmente está trabajando en brindar a sus clientes "soluciones técnicas integrales" para el sector energético, por lo que es reconocida en este ámbito por sus capacidades productivas basadas en la innovación tecnológica, alto valor agregado y procesos sostenibles.

\section{Agradecimiento:}

Quisiéramos expresar nuestro profundo agradecimiento a la psicóloga organizacional Michelle Mejía Occaña, que fue alumna destacada de la UISEK, por sus aportes significativos a este artículo.

\section{Referencias bibliográficas}

Agudo, F. (1998). Comportamiento Humano. McGraw-Hill Latinoamericana. México.

Equipo Vértice. (2010). Gestión de la calidad (ISO 9001/2008). Editorial Vértice.

Davenport, T. (2000). Capital Humano. Creando ventajas competitivas a través de las personas. Ediciones Gestión 2000, S.A. España. pp: 44-49 
Calavia, M. (2011). Aplicación de la norma ISO 9001 para la mejora de la Gestión : el caso de la Dirección del Sistema Nacional de Capacitación del Instituto Nacional de Administración Pública- Recuperado en Abril 2011, de https://aaeap.org.ar/wpcontent/uploads/2013/6cong/CALAVIA_MARCELO.pdf

Chiavenato, I. (2006) Introducción a la Teoría General de la Administración del Talento Humano. McGraw Hill Interamericana editores S.A. Séptima Edición. México.

Chiavenato, I. (2007). Administración de Recursos Humanos. México: McGraw-Hill.

Deschamps, G. (1998) Cómo rentabilizar la inversión en Formación, Editorial Gestión y planificación integral, S.A., España.

Fernández, C., Ramírez, M., \& Díaz V. (2008). La sinergia y los efectos ignorados de la capacitación: el impacto sistémico en los resultados de las organizaciones turísticas. Retos Turísticos. Cuba.

González, M., \& Olivares, S. (2014). Planeación e integración de los recursos humanos: Capital humano (2a. ed.). México.

Hernández, C. E. E., \& Molina, A. A. T. (2004). Una alternativa para la evaluación del impacto de la capacitación en las organizaciones empresariales. Folletos gerenciales, 12(5) : 16-25, 2008.

Rincón, R. D. (2002). Modelo para la implementación de un sistema de gestión de la calidad basado en la norma ISO 9001. Revista Universidad EAFIT, 38(126), 4755.

Sáez, F. Druker, P. (2017) Sobre la gestión personal Productividad y GTD, recuperado en 2017, de: https://facilethings.com/blog/es/peter-drucker-self-management.

Rodríguez, E. M., \& Ramírez-Buendía, P. (1991). Administración de la capacitación. Serie Capacitación efectiva. Editorial. McGraw-Hill.

Santillán, W. (2017). Nuevas prácticas para mejorar el clima laboral. Quito: Don Bosco.

Taylor, F. (1981) Principios de Administración Científica, El Ateneo, Buenos Aires.

Werther, W., Keith, D. (2000). Administración de Personal y Recursos Humanos. México: Quinta edición. Mc Graw Hill. 48-225

Wurgaft, J. (1998). La capacitación laboral en los países andinos. Lima, Perú: Organización Internacional del Trabajo.

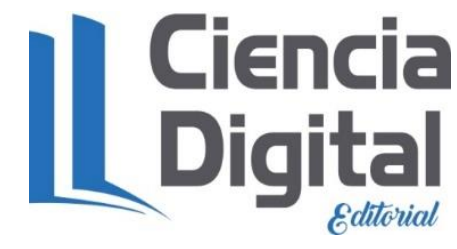




\section{PARA CITAR EL ARTÍCULO INDEXADO.}

Santillán Marroquín, W. E., Araque Salazar, M. C., \& Guanochanga Oña, D. (2021). La norma ISO 9001 en el proceso de capacitación en una compañía de servicios petroleros. ConcienciaDigital, 4(2.1), 35-51. https://doi.org/10.33262/concienciadigital.v4i2.1.1705

\section{Ciencia}

El artículo que se publica es de exclusiva responsabilidad de los autores y no necesariamente reflejan el pensamiento de la Revista Ciencia Digital.

El artículo queda en propiedad de la revista y, por tanto, su publicación parcial y/o total en otro medio tiene que ser autorizado por el director de la Revista Ciencia Digital.

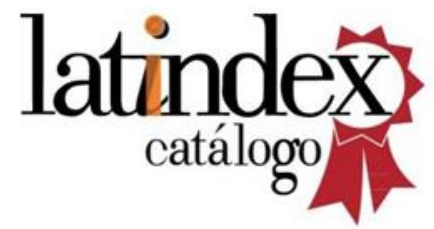

